\title{
Immediate Effects of Flexi-bar Exercise With Knee Push-up Plus on Shoulder Joint Position Sense and Muscle Activity in Subjects With Scapular Winging
}

\author{
Seok-hyun Kim¹, PT, BPT, Heon-seock Cynn¹, PT, PhD, Seung-min Baik ${ }^{1,2}$, PT, BPT \\ ${ }^{1}$ Applied Kinesiology and Ergonomic Technology Laboratory, Department of Physical Therapy, The Graduate School, Yonsei University, \\ Wonju, ${ }^{2}$ Department of Physical Therapy, Division of Health Science, Baekseok University, Cheonan, Korea
}

\author{
Article Info \\ Received September 17, 2021 \\ Revised October 2, 2021 \\ Accepted October 6, 2021 \\ Corresponding Author \\ Heon-seock Cynn \\ E-mail: cynn@yonsei.ac.kr \\ https://orcid.org/0000-0002-5810-2371
}

\section{Key Words}

Flexi-bar

Joint position sense

Knee push-up plus

Lower trapezius

Scapular winging

Serratus anterior
Background: Individuals with scapular winging may have proprioceptive dysfunction which is important for motor control and causes shoulder instability. Reduced serratus anterior (SA) and lower trapezius (LT) muscle activity accompanied by over-active upper trapezius (UT), and pectoralis major (PM) may be contributing factors. Flexi-bar (FB) exercise may be used to increase joint position sense (JPS) and alter the target muscle activities.

Objects: This study aimed to investigate the immediate effects of flexi-bar exercise prior to knee push-up plus (FPK) versus knee push-up plus (KPP) on JPS and muscle activity of SA, LT, UT, and PM in subjects with scapular winging.

Methods: Eighteen subjects with scapular winging were recruited. JPS was investigated at baseline, after KPP and after FPK. Passive and active JPS errors were calculated by isokinetic equipment. Surface electromyography was used to record muscle activities during KPP and FPK. One-way repeated-measures analysis of variance and post hoc analyses were used to analyze the JPS error measured at baseline, after KPP and after FPK. Paired t-tests were used to compare muscle activities between KPP and FPK.

Results: Passive JPS error was significantly decreased after KPP ( $p=0.005)$ and after FPK ( $p$ $=0.003$ ) compared to the baseline. Active JPS error was also significantly decreased after KPP $(p=0.016)$ and after FPK ( $p=0.012)$ compared to the baseline. There was no significant difference in the passive and active JPS errors between KPP and FPK. SA activity during FPK was significantly increased ( $p=0.024)$, and LT activity during FPK was significantly increased ( $p=$ 0.006). There were no significant differences in the UT and PM activity.

Conclusion: FB might be recommended to immediately improve passive and active JPS and to selectively increase SA and LT muscle activities during KPP in individuals with scapular winging.

\section{INTRODUCTION}

Scapular dyskinesis is the term used to describe changes in the positioning or movement of the scapula and is associated with changes in the activation of the scapular muscles [1]. There are several mechanisms that may lead to scapular dyskinesis including postural imbalance, scapular muscle imbalance or weakness and proprioceptive dysfunctions [2]. Scapular winging, a type of scapular dyskinesis, is defined as an increased prominence of the whole medial border of the scapula that increases the internal rotation of the scapula [3]. Especially, weakness of the serratus anterior (SA) muscle without long thoracic nerve injury is an important cause of scapula winging [4]. Scapular winging affects glenohumeral instability and causes functional impairment [5].

Many researchers have investigated various exercises for increasing SA activity [6]. Of these exercises, the push-up plus elicits the highest average electromyography (EMG) activity of the SA, and the lowest EMG activity of the upper trapezius (UT) compared to other SA exercises such as wall slide, scaption and dynamic hug [7,8]. Especially, several studies have suggested the knee push-up plus (KPP) for individuals in the early rehabilitation because KPP limits the amount of weight bearing and demonstrates higher SA activity compared to the standard push-up plus [6,9]. In addition, as a closed kinetic exercise, the KPP exercise can be beneficial for dynamic sta- 
bilization and stimulating proprioception in the shoulder complex $[6,10,11]$.

However, individuals with scapular winging should be careful about compensation from other synergist muscles during upper extremity movements. Specifically, reduced SA and lower trapezius (LT) activation accompanied by over-activation of the UT which are contributing factors for abnormal scapular motion during arm elevation [12]. Additionally, a previous study demonstrated that individuals with scapular winging showed increased pectoralis major (PM) activity and decreased SA activity during protraction [13]. Excessive PM activation may compensate for a weak SA leading to abnormal scapular motion and shoulder pathologies such as shoulder impingement and shoulder anterior joint dislocations $[13,14]$.

Shoulder proprioception is also negatively affected by scapular dyskinesis [2]. Joint position sense (JPS), a type of proprioception, is especially important for shoulder function because the stability of shoulder joint is sacrificed for large range of motion [15]. A deficit in proprioception might affect neuromuscular dysfunction and is associated with shoulder instability and impingement [16,17]. Therefore, proprioceptive exercises have been recommended in individuals with shoulder instability [18].

Among the training exercises for proprioception, the flexibar (FB), an vibratory device capable of generating a low-frequency (about $4.5 \mathrm{~Hz}$ ) vibration with manual shaking has been used to facilitate the tonic vibration reflex (TVR) in a short time [19,20]. During TVR, alpha motor neuron excitation occurs which promote immediate and transient improvement in the neuromuscular performance, increased muscle activity, and proprioception [21-23]. FB exercise increased shoulder muscle activity immediately compared to dumbbells and elastic bands and, increased muscle activity was related to the improvement of JPS [24-26]. Thus, FB might be an effective exercise tool for promoting scapular stability and proprioceptive training.

However, no previous studies have investigated the immediate effects of FB prior to KPP (FPK) versus KPP on JPS and muscle activities in subjects with scapular winging. Therefore, the purpose of this study was to compare the immediate effects FPK versus KPP on JPS and muscle activity of SA, LT, UT and PM in subjects with scapular winging. Our hypothesis was that the JPS would be different at the baseline, after KPP and after FPK, and the muscle activity of SA, LT, UT and PM would be different during FPK compared to KPP in subjects with scapular winging.

\section{MATERIALS AND METHODS}

\section{Subjects}

G-power software (ver. 3.1.9.2; Franz Faul, Kiel University, Kiel, Germany) was used to conduct the power analyses. Eighteen subjects with scapular winging were recruited. A sample size of 18 was obtained from a pilot study to achieve a power of 0.80 and an effect size of 0.62 with an alpha level of 0.05 . The demographic data are shown in Table 1.

Individuals were considered eligible if they had scapular winging which was measured by using a scapulometer. If the medial border of the scapula protruded by a distance $\geq 2 \mathrm{~cm}$ from the posterior thoracic rib cage in a quadruped position, the scapula was considered to have winging [9]. The scapulometer was positioned on the $\mathrm{T} 5$ spinous process and measurement was performed between the scapular medial border and spinous process. The Intraclass correlation coefficient (ICC) of the scapulometer in a previous study was 0.99 (95\% confidence interval 0.98-0.99, standard error of measurement $0.51 \mathrm{~mm}$, and minimum detectable change $1.41 \mathrm{~mm}$ ). The exclusion criteria were a previous history of shoulder surgery or trauma, such as physical injuries caused by an external force and stroke, epilepsy, multiple sclerosis, Parkinson's disease, or peripheral neuropathy. Additional exclusion criteria were lateral or medial epicondylitis, carpal tunnel syndrome, tendinopathies, or upper-extremity problems such as shoulder tendinitis, impingement, or pain due to difficulty in performing the exercise in this study [27,28]. individuals who could not perform KPP without discomfort were excluded [29].

All subjects were informed of the experimental protocol, and they gave written consent for study participantion. The experimental protocol was approved by the Yonsei University Mirae Campus Institutional Review Board (approval number 1041849-202104-BM-061-02).

Table 1. Descriptive characteristics of the subjects ( $N=18)$

\begin{tabular}{lc}
\hline \multicolumn{1}{c}{ Variables } & Values \\
\hline Age $(\mathrm{y})$ & $26.2 \pm 2.04$ \\
Height $(\mathrm{cm})$ & $174 \pm 5.55$ \\
Weight $(\mathrm{kg})$ & $76.65 \pm 12.09$ \\
Body mass index $\left(\mathrm{kg} / \mathrm{m}^{2}\right)$ & $25.09 \pm 3.45$ \\
Magnitude of scapular winging $(\mathrm{cm})$ & $2.88 \pm 0.70$ \\
\hline
\end{tabular}

Values are presented as mean \pm standard deviation. 


\section{EMG Recording and Data Processing}

To investigate the muscle activity of SA, LT, UT and PM, surface EMG (Noraxon, Inc, Scottsdale, AZ, USA) with a MyoResearch Master Edition software package (ver. 3.16; Noraxon Inc, Scottsdale, AZ, USA) was used [9]. EMG data were sampled at $1,500 \mathrm{~Hz}$, overall gain of $500, \mathrm{CMRR}>100 \mathrm{~dB}$, and input impedance $>100$ Mohm. A digital band-pass filter, between 20 and $450 \mathrm{~Hz}$, filtered the raw signals, along with notch filters $(60 \mathrm{~Hz})$. The raw data were processed into the root mean square (RMS). RMS were enveloped with window size of 200 milliseconds before being recorded. After shaving the hair and scrubbing the skin with alcohol, the principal investigator (PI) attached disposable $\mathrm{Ag} / \mathrm{AgCl}$ surface electrodes on the subject's muscle belly [9]. The electrodes were positioned over the midsection of the subject's muscle belly parallel to the target muscle fibers on the greater winging side. Two electrodes were placed about $2 \mathrm{~cm}$ apart in the direction of the muscle fibers to minimize cross-talk. For the SA, two active electrodes were placed horizontally ( $2 \mathrm{~cm}$ apart) just below the axillary area, at the level of the inferior tip of the scapula, and just medial of the latissimus dorsi. For the LT, two active electrodes $(2 \mathrm{~cm}$ apart) were placed next to the medial edge of the scapula at a $55^{\circ}$ oblique angle. For the UT, two active electrodes were placed along the ridge of the shoulder, slightly lateral to and one-half the distance between the cervical spine at C7 and the acromion. For PM (sternal fiber), two active electrodes were placed on the chest wall horizontal to the arising muscle mass $(2 \mathrm{~cm}$ from the axillary fold) [30]. The EMG activity of the SA, LT, UT and PM muscles was recorded when the subjects held a protracted position during KPP and FPK. The last three trials of the final KPP set were used for data analysis. The EMG amplitudes of the SA, LT, UT, and PM muscles were represented as a percentage of the reference voluntary contraction (RVC) value. To collect the RVC data, the subjects flexed their shoulder by $90^{\circ}$ in the scapular plane for three trials of 5 seconds each while wearing a 6.5 $\mathrm{kg}$ sandbag on their forearm. The EMG signal collected during each exercise was expressed as a percentage of the calculated root mean square of RVC (\%RVC) [27].

\section{Measurement of the Passive and Active Joint Position Sense Errors}

Passive and active JPS errors were measured by isokinetic equipment (Biodex System 4 pro dynamometer, Biodex Medical systems Inc., Shirley, NY, USA) through reproduction of passive and active position. The subjects were instructed to close their eyes. To begin passive and active JPS error measurements, the subjects were asked to sit on the isokinetic equipment with $90^{\circ}$ shoulder abduction, in $90^{\circ}$ external rotation, and $90^{\circ}$ elbow flexion [16,31]. Passive and active JPS errors were calculated while the shoulder was internally rotated from $90^{\circ}$ external rotation to the target angle of $0^{\circ}$ because a previous systematic review study reported that an internal rotation (IR) protocol resulted in a strong weighted average both intrasession and intersession reliabilities; an ICC of 0.88 $\pm 0.01(n=53$, intrasession) and $0.98 \pm 0.01$ ( $n=31$, intersession) [17]. The isokinetic equipment moved the arm by the rate of $5^{\circ} / \mathrm{s}$ and held the arm at a target angle of $0^{\circ}$ for 10 seconds so that the subejects can recognize this target angle of $0^{\circ}$. The subjects were asked to push the hold button and stop the isokinetic equipment when they felt that the arm had reached the target angle of $0^{\circ}$ by being passively moved by the isokinetic equipment. The passive JPS error was calculated as the difference between the target angle and the angle marked passively by the subjects. For active JPS, the subjects followed the same procedure to get accustomed to the target angle of $0^{\circ}$ with the isokinetic equipment. Then, they were asked to find the target angle of $0^{\circ}$ by moving the isokinetic equipment actively themselves. The active JPS error was calculated as the difference between the target angle and the angle marked actively by the subjects. Measurements were repeated four times and the mean value of the passive and active angular errors was used for the statistical analysis [31,32]. Passive and active JPS errors were recorded at the baseline, after KPP, and after FPK.

\section{Knee Push-up Plus}

For the KPP's start position, the subjects placed both hands and knees on the ground, shoulder-width apart as in a quadruped position. The elbows were fully extended with shoulder flexion of $110^{\circ}$ and knees were flexed $90^{\circ}$ to the ground (Figure 1) [33]. Next, a full forward scapular protraction (the "plus") was performed until the thoracic vertebrae touched a wooden target bar. This position was held for 5 seconds before the starting position was resumed. To standardize and guide KPP, a wooden target bar was installed at the mid-point achieved by the fourth thoracic vertebrae during the "plus" phase. The height of the wooden target bar was within the limits attainable with normal thoracic kyphosis. Additionally, two white pillars were placed in front of the shoulders to guide the "plus 


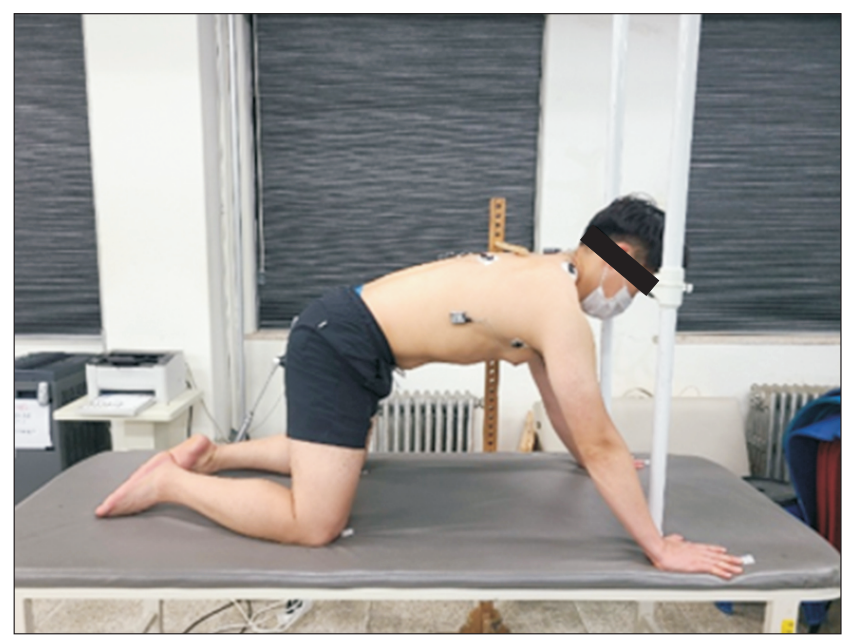

Figure 1. Knee push-up plus exercise.

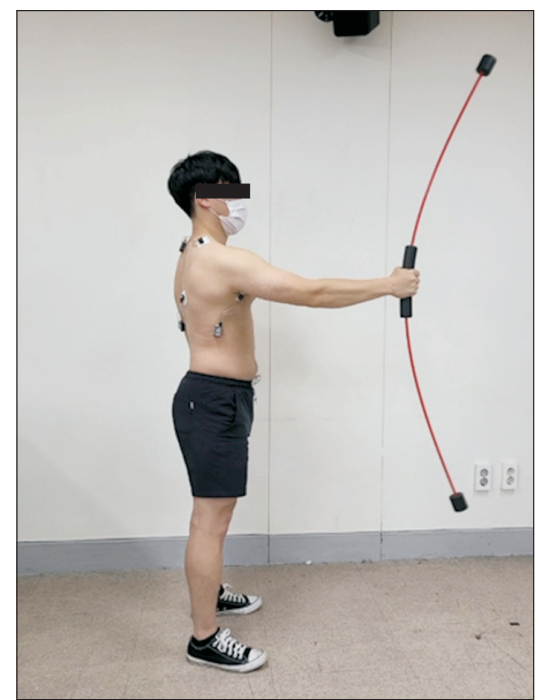

Figure 2. Flexi-bar exercise.

motion" and limit the lateral movement when a subject assumed a push-up posture. Three sets of 10 repetitions of KPP were performed [34]. To minimize muscle fatigue, a 3-minute resting period was allowed between each set [9,10,29,35].

\section{Flexi-bar Exercise}

The FB exercise was performed in a standing position with the feet parallel and shoulder-width apart, holding the FB vertically and perpendicular to the ground. The subjects maintained with the position with the elbows in slight flexion (approximately $30^{\circ}$ ) and the shoulders in $90^{\circ}$ flexion against the vibration of the FB. FB exercise was performed using FLEXIBAR $^{\circledR}$ Standard (REDBALANCE, Seoul, Korea) that was 1.6$\mathrm{m}$ long, weighed $0.65 \mathrm{~g}$, and was composed of fiberglass and

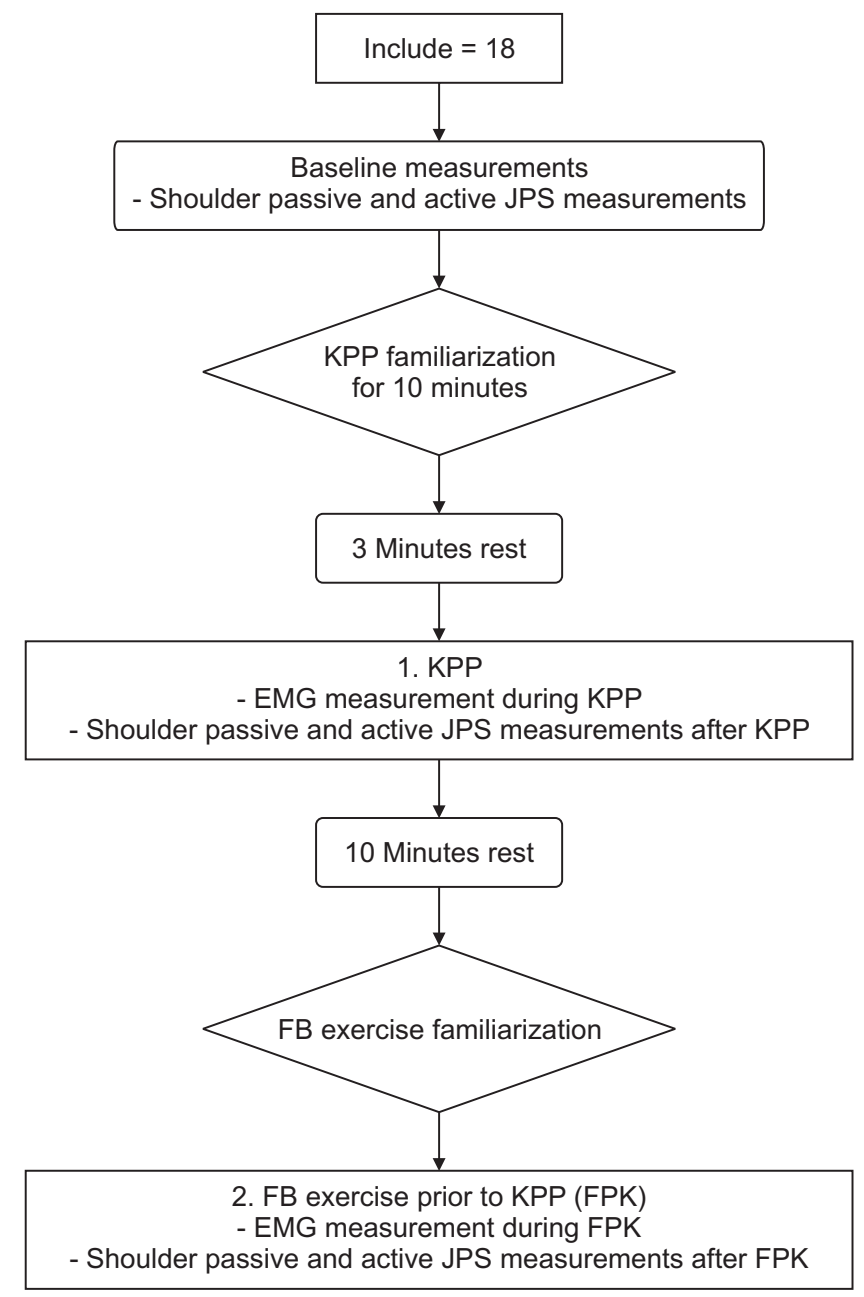

Figure 3. Flow chart of the recruitment of study subjects, measurements and exercise protocol. JPS, joint position sense; KPP, knee push-up plus; EMG, electromyography; FB, flexi-bar exercise; FPK, flexi-bar exercise prior to knee push-up plus.

rubber (Figure 2) [28]. Bar oscillation was generated in the sagittal plane with anteroposterior movements, and resulted in an oscillation frequency of $4.5 \mathrm{~Hz}[19,24,28,36]$. The rhythm of the pole movements was controlled by a metronome set at 270 bpm [19]. Anteroposterior oscillation was chosen because the subjects considered it easy to maintain the required frequency and it was less provocative for volunteers in a previous study. Three attempts with a duration of 12 seconds and with a 1-minute rest between the attempts were performed because, pilot studies have indicated that untrained subjects begin to experience fatigue at approximately 15 seconds [28].

\section{Procedure}

Every subject measured the JPS error at baseline. Then, they were educated about how to perform KPP, and were familiar- 
ized for approximately 10 mintues by the PI and were allowed a 3-minute rest before the testing protocol to minimize muscle fatigue. The subjects performed three sets of 10 repetitions of KPP. EMG amplitude was assessed during KPP and JPS errors were measured after KPP. A 10-minute rest was provided to minimize muscle fatigue before the second testing session. Next, the subjects were familiarized with the FB exercise so that they could perform it correctly. The subjects performed three attempts of FB exercise and then, performed three sets of 10 repetitions of KPP. The same procedures described previously was executed to assess the EMG amplitude and JPS error of FPK (Figure 3).

\section{Statistical Analysis}

The SPSS Statistics 26 software (IBM Co., Armonk, NY, USA) was used to perform all statistical analyses. Shapiro-Wilk tests was conducted to confirm the assumption of normal distribution. A one-way, repeated-measures analysis of variance (ANOVA) was used to assess the statistical significance of a change in the JPS error measured at the baseline, after KPP and after FPK. Paired t-tests were used to assess the statistical significance of a change in the muscle activity of SA, LT, UT, and PM between KPP and FPK. Level of significance was set at $p<0.05$. If a significant differences was found on performing a one-way repeated-measures ANOVA, post hoc test with Bonferroni correction $(0.05 / 3)$ was performed [37].

\section{RESULTS}

There was a significant difference in the passive JPS error (F $=11.354, \mathrm{p}=0.000, \mathrm{ES}=0.81$ ) measured at the baseline, after KPP and after FPK. In the post hoc test with Bonferroni correction, the passive JPS error was significantly decreased after $\operatorname{KPP}(p=0.005, E S=0.79)$ and after FPK $(p=0.003, E S=1.05)$ compared to the baseline. There was no significant difference in the passive JPS error between KPP and FPK ( $p=1.000$, ES = 0.23) (Table 2, Figure 4).

In addition, there was significant difference in the active JPS error $(F=7.802, p=0.002, E S=0.67)$ measured at the baseline, after KPP and after FPK. In the post hoc test with Bonferroni correction, the active JPS error was significantly decreased after $\operatorname{KPP}(p=0.016, E S=0.65)$ and after FPK ( $=0.012$, ES = 0.80) compared to the baseline. There was no significant difference in the active JPS error between KPP and FPK ( $p=1.000$, $\mathrm{ES}=0.17)$ (Table 2, Figure 4).

The muscle activity of SA significantly increased during FPK

Table 2. Data of mean \pm standard deviation values of shoulder passive and active JPS error measured at baseline, after KPP and after FPK

\begin{tabular}{lcccrr}
\hline \multirow{2}{*}{ Variables } & & JPS error & F & \\
\cline { 2 - 4 } & Baseline & After KPP & After FPK & & \\
\hline Passive JPS & $7.64 \pm 3.17$ & $5.29 \pm 2.70^{* *}$ & $4.73 \pm 1.96^{* *}$ & 11.354 & $0.000^{*}$ \\
Active JPS & $5.56 \pm 2.66$ & $3.99 \pm 1.80^{* *}$ & $3.68 \pm 1.78^{* *}$ & 7.802 & $0.002^{*}$ \\
\hline
\end{tabular}

JPS, joint position sense; KPP, knee push-up plus; FPK, flexi-bar exercise prior to knee push-up plus. *Indicates a significant difference (p < 0.05$). * *$ Indicates significant difference compared to the baseline $(p<0.05 / 3)$.
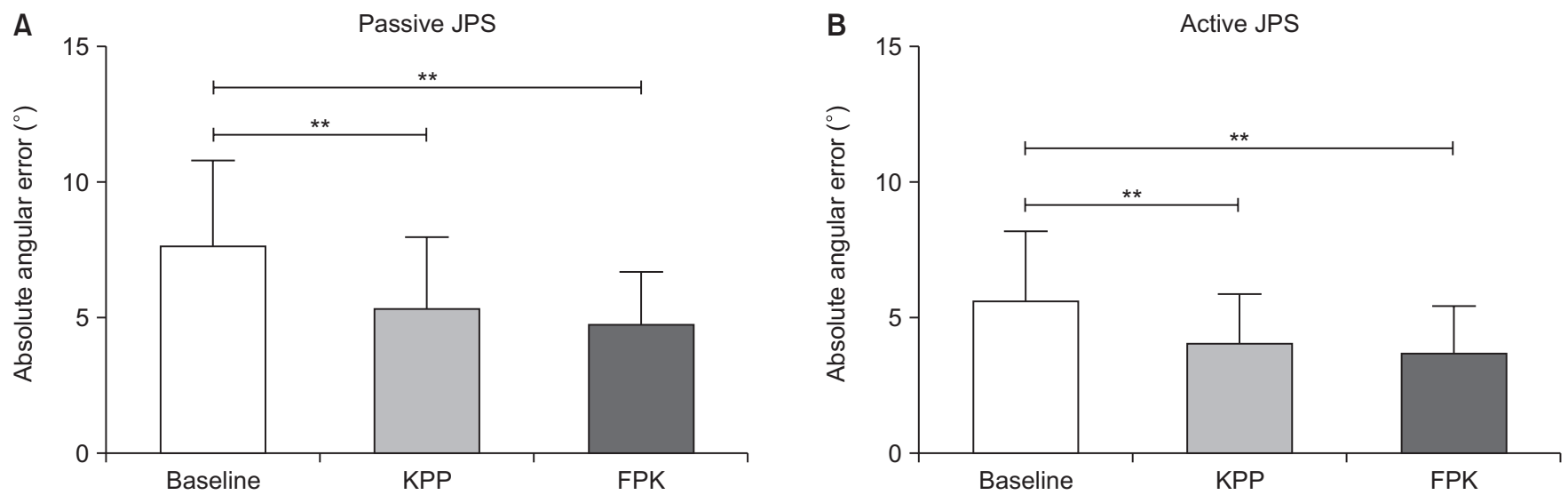

Figure 4. Data of (A) passive and (B) active JPS errors measured at baseline, after KPP and after FPK. JPS, joint position sense; KPP, knee push-up plus; FPK, flexi-bar exercise prior to knee push-up plus. **Significant difference by Bonferroni adjustment $(p<0.05 / 3)$. 
compared to $\mathrm{KPP}(\mathrm{t}=-2.482, \mathrm{p}=0.024, \mathrm{ES}=0.38)$. The activity of LT also significantly increased during FPK compared to KPP $(\mathrm{t}=-3.127, \mathrm{p}=0.006, \mathrm{ES}=0.29)$. However, there was no significant difference in the activity of UT between KPP and FPK (t $=-1.034, \mathrm{p}=0.315, \mathrm{ES}=0.06$ ). Additionally, there was no significant difference in the activity of PM between KPP and FPK $(\mathrm{t}$ $=1.147, \mathrm{p}=0.267, \mathrm{ES}=0.13)($ Table 3, Figures 5, 6).

\section{DISCUSSION}

The purpose of this study was to identify the immediate effects of FPK versus KPP on JPS and muscle activities in subjects with scapular winging. We hypothesized that the JPS would be different among the baseline, after KPP and after FPK measurements, and the muscle activity of SA, LT, UT, and PM during FPK would show an immediate change compared to KPP.

Table 3. Data of mean \pm standard deviation values of muscle activity $(\% \mathrm{RVC})$ during KPP and FPK

\begin{tabular}{lcccc}
\hline \multirow{2}{*}{ Variables } & \multicolumn{2}{c}{ Muscle activity (\%RVC) } & $\mathrm{t}$ & $\mathrm{p}$-value \\
\cline { 2 - 4 } & KPP & FPK & -2.482 & $0.024^{*}$ \\
Serratus anterior & $70.36 \pm 59.08$ & $96.96 \pm 76.06$ & -3.127 & $0.006^{*}$ \\
Lower trapezius & $14.46 \pm 11.47$ & $18.24 \pm 14.30$ & -1.034 & 0.315 \\
Upper trapezius & $17.04 \pm 12.95$ & $17.83 \pm 12.83$ & 1.147 & 0.267 \\
Pectoralis major & $46.69 \pm 66.64$ & $38.78 \pm 43.84$ & & \\
\hline
\end{tabular}

RVC, reference voluntary contraction; KPP, knee push-up plus; FPK, flexi-bar exercise prior to knee push-up plus. ${ }^{*}$ Indicates a significant difference lp < 0.05).

A

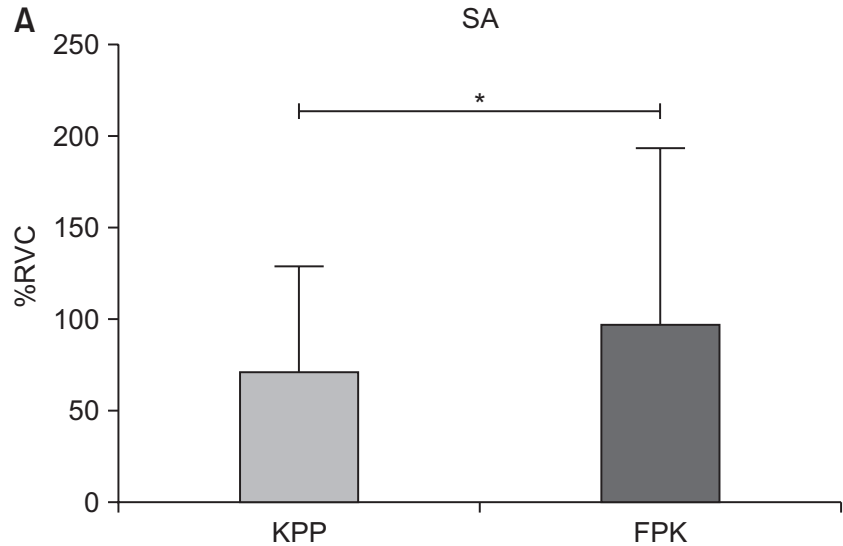

B

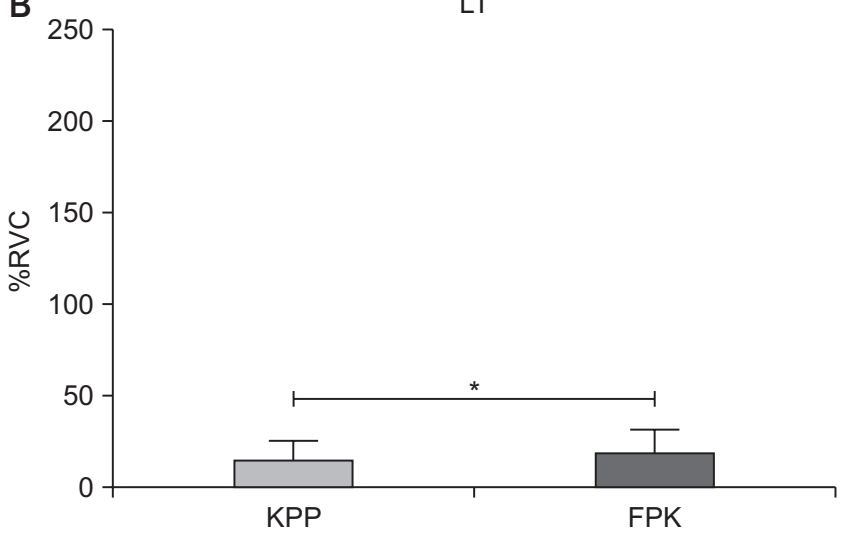

Figure 5. Data of \%RVC of (A) SA and (B) LT between KPP and FPK. RVC, reference voluntary contraction; SA, serratus anterior; LT, lower trapezius; KPP, knee push-up plus; FPK, flexi-bar exercise prior to knee push-up plus. *Significant difference $(p<0.05)$.

A

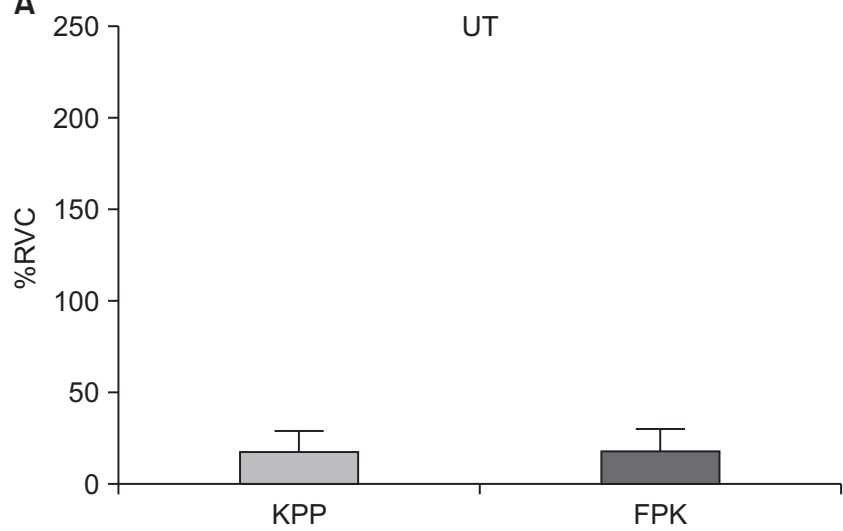

B

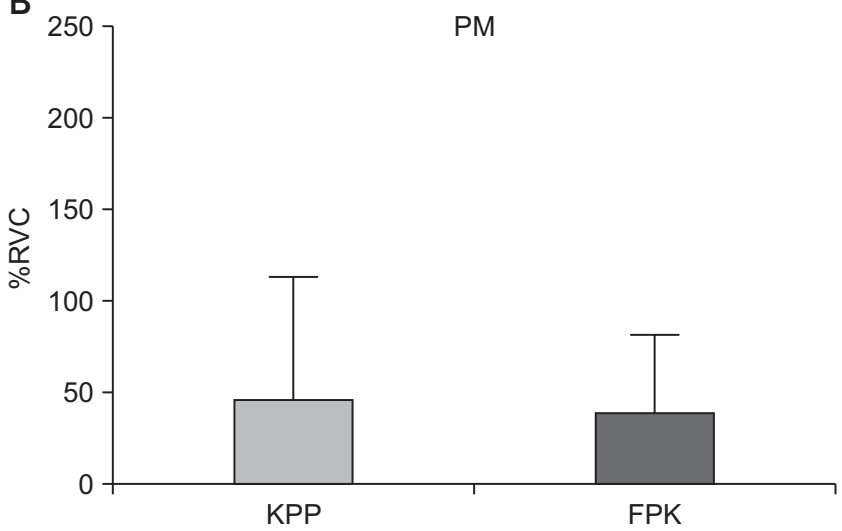

Figure 6. Data of \%RVC of (A) UT and (B) PM between KPP and FPK. RVC, reference voluntary contraction; UT, upper trapezius; PM, pectoralis major; KPP, knee push-up plus; FPK, flexi-bar exercise prior to knee push-up plus. 
Our results partially supported our hypothesis. The results indicate that both KPP and FPK decrease the passive and active JPS errors immediately, and FPK increases SA and LT activity compared to KPP.

In this study, passive and active JPS errors were significantly decreased immediately after KPP by $30.75 \%$ and $28.23 \%$, respectively, compared to the baseline. The findings of this study are in agreement with those of a previous study by Daman et al. [38]. Daman et al. [38] demonstrated that knee joint proprioception was improved immediately after closed kinetic chain exercise. Closed kinetic chain exercises affected the integration of proprioceptors, specifically Ruffini's endings, $\mathrm{Pa}$ cinian corpuscles, Golgi-Tendon Organs, and muscle spindles [39]. KPP as a closed kinetic chain exercise, stimulats proprioceptive receptors $[38,39]$. Due to the sensitive mechanoreceptors that respond to changes in the pressure of the articular capsule by axial loading and joint compression, KPP, as a form of closed kinetic chain exercise, improves JPS.

Similarly, passive and active JPS errors were significantly decreased after FPK by $38.08 \%$ and $33.81 \%$ respectively, compared to the baseline. This finding is the first evidence that both passive and active JPS errors may be decreased immediately after FPK in subjects with scapular winging. These results are in agreement with those of a previous study by Schulte and Warner [40] that used FB. It was considered that the mechanism for improved JPS was that vibration stimuli from FB increased joint stability by activation of the joint mechanoreceptors, and stimulated alpha and gamma motor neurons related to improved JPS [16,28]. FB necessitates neuromuscular control and proprioceptive feedback to optimize a rhythmic pattern of alternating contraction between the agonist and antagonist muscles [40]. Therefore, FPK as a form of vibration and closed kinetic chain exercise contributed to improvement in passive and active JPS in this study.

Interestingly, there were no significant JPS error differences between KPP and FPK. To our best knowledge, no previous studies have reported this finding in the literature. The position and application of FB used in this study may be the reason for this finding. FB exercise mainly affects the muscle spindles, but ligaments and joint capsules are also important to JPS. To stimulate the ligaments and joint capsules, the full range of motion and fast motion in the end range of the joint are required [41]. In contrast, in our study, FB exercise was performed while maintaining $90^{\circ}$ shoulder flexion in the mid range of joint. Thus, a significant JPS error difference may not have been detected between KPP and FPK.

Compared to KPP, SA activity in FPK was significantly increased by $40.41 \%$. Previous studies have reported that exercise using FB increased SA activation compared to Theraband or, non-oscillatory poles $[19,25]$. This finding indicates that FB exercise increases muscle activity of SA by increasing neuromuscular facilitation by TVR. FB has been hypothesized to strengthen the shoulder stabilizing muscle by training the contractile tissues directly, and also indirectly by affecting the joint and surrounding non-contractile tissues [42]. A previous study demonstrated that FB exercises in the frontal plane with mediolateral or superoinferior oscillations demonstrated lower SA activation than in the sagittal plane with anteroposterior oscillation. Specifically, FB exercise with anteroposterior oscillation in the sagittal plane is required to rapidly alternate between protraction and retraction of the scapula which creates equilibrium between stability and mobility of the scapula. This suggets that repetitive protraction and retraction caused by FB provided adequate resistance for SA pre-activation and increases muscle activity of SA during FPK.

Compared to KPP, LT activity in FPK was significantly increased by $27.19 \%$. Several studies have revealed that FB exercise increases LT activity $[19,25,36]$. They demonstrated that FB exercise provideds an adequate task in a short amount of time to promote pre-activation of LT activity during FPK. LT contributed to scapular upward rotation, retraction, and posterior tilt during humeral elevation. During FB with anteroposterior oscillation in the sagittal plane, LT is activated not only to maintain the arm in $90^{\circ}$ shoulder flexion but also to repeatedly perform protraction and retraction along with SA. To maintain this position, the subjects contracted their arms and shoulders against the vibration and FB weight. Particularly, when the subjects pushed the FB to the distal side, the FB returned to the proximal side due to inertia, and simultaneously LT and SA were contracted eccentrically and concentrically, respectively, to withstand the FB resistance.

UT muscle activity during FPK was not significantly different from that during KPP. This finding was inconsistent with a study of Park et al. [43], which reported that KPP with vibration decreased UT activity in subjects with scapular winging. The discrepancy between the study by Park et al. [43] and our study was that they used whole body vibration (WBV) located on the floor during KPP. Vibration stimuli, which were 
passively provided from WBV, increased SA activity through TVR and decreasesd UT activity during KPP. As synergists for scapular stabilization, increased SA activity might have caused decreased excessive muscle activity and compenstation of UT during KPP because the synergistic muscles might affect each other through movement $[14,44]$. Contrastingly, in our study, FB exercise was performed actively in the standing position maintaining scapular upward rotation and shoulder flexion against gravity while holding the FB in hand. FB weight along with additional load from oscillation may have promoted UT muscle recruitment. In fact, FB vibration may increase the gravitational load. FB exercise was found to immediately increase motor unit recruitment and activity of the adjacent muscles according to the increased load [25].

PM activity in FPK was not significantly different from KPP. Park et al. [14] reported that increased SA activity may be related to decreased PM acitivty during wall push-up plus with isometric horizontal abduction. Because, when the movement is executed in the same range, decreased activity of one muscle increases activity of another muscle activity [14]. However, FB exercise is inherently a dynamic exercise compared to wall push-up plus as well as shoulder flexion [27]. Parry et al. [24] compared the effect of FB and 10 pound dumbbells exercise, and showed that FB exercise elicited greater peak PM activity which exceed $50 \%$ of the maximal voluntary isometric contraction compared to 10 pound dumbbell. Even though the weight of FB was much lower than the 10 pound dumbbell, more PM recruitment was required from repetitive protraction during the FB exercise.

This study had some limitations. First, the study findings may not be generalizabled to other populations because the subjects were young individuals with scapular winging. Second, the activity of middle trapezius, which is important for scapular stabilization, was not recorded during KPP. Third, the order of KPP and FPK was not randomized. However, we considerd that performing KPP first may reduce pontential carry over effect or learning effect. Fourth, cross talk could occur with possible interference during surface EMG data collection. Further, longitudinal studies of FB application in various postures are warranted in subjects with different pathologies.

\section{CONCLUSIONS}

The immediate effects of FPK versus KPP on JPS error and muscles activity in subjects with scapular winging were investigated in this study. Our results demonstrated that the passive and active JPS error weres significantly decreased immediately after KPP as well as after FPK compared to the baseline. Muscle activity of SA and LT during FPK was significantly increased compared to KPP. Therefore, FB might be useful to improve JPS, and selectively increase SA and LT muscle activity during KPP in individuals with scapular winging.

\section{CONFLICTS OF INTEREST}

No potential conflict of interest relevant to this article was reported.

\section{AUTHOR CONTRIBUTIONS}

Conceptualization: SK, HC, SB. Data curation: SK, SB. Formal analysis: SK. Investigation: SK, HC, SB. Methodology: SK, HC, SB. Project administration: SK, HC, SB. Resources: SK, HC, SB. Supervision: SK, HC, SB. Visualization: SK, SB. Writing original draft: SK. Writing - review \& editing: SK, HC, SB.

\section{ORCID}

Seok-hyun Kim, https://orcid.org/0000-0002-0951-0360

Seung-min Baik, https://orcid.org/0000-0001-7885-9374

\section{REFERENCES}

1. de Araújo RC, Pirauá ALT, Beltrão NB, Pitangui ACR. Activity of periscapular muscles and its correlation with external oblique during push-up: does scapular dyskinesis change the electromyographic response? J Sports Sci 2018;36(5):571-7.

2. Ishita S, Siddhartha S, Umer A. Effect of electrical muscle stimulation with voluntary contraction and taping on joint position sense in asymptomatic scapular dyskinesic patients. Int J Phys Med Rehabil 2014;2(2):1000190.

3. Kibler WB, Sciascia A. Current concepts: scapular dyskinesis. Br J Sports Med 2010;44(5):300-5.

4. Marín PJ, Santos-Lozano A, Santin-Medeiros F, Vicente-Rodriguez G, Casajús JA, Hazell TJ, et al. Whole-body vibration increases upper and lower body muscle activity in older adults: potential use of vibration accessories. J Electromyogr Kinesiol 2012;22(3):456-62. 
5. Mueller AM, Entezari V, Rosso C, McKenzie B, Hasebrock A, Cereatti A, et al. The effect of simulated scapular winging on glenohumeral joint translations. J Shoulder Elbow Surg 2013;22(7):986-92

6. Ludewig PM, Hoff MS, Osowski EE, Meschke SA, Rundquist PJ. Relative balance of serratus anterior and upper trapezius muscle activity during push-up exercises. Am J Sports Med 2004;32(2):484-93

7. Hardwick DH, Beebe JA, McDonnell MK, Lang CE. A comparison of serratus anterior muscle activation during a wall slide exercise and other traditional exercises. J Orthop Sports Phys Ther 2006;36(12):903-10.

8. Decker MJ, Hintermeister RA, Faber KJ, Hawkins RJ. Serratus anterior muscle activity during selected rehabilitation exercises. Am J Sports Med 1999;27(6):784-91.

9. Shin AR, Lee JH, Kim DE, Cynn HS. Thera-Band application changes muscle activity and kyphosis and scapular winging during knee push-up plus in subjects with scapular winging: the cross-sectional study. Medicine (Baltimore) 2018;97(14):e0348.

10. Kang FJ, Ou HL, Lin KY, Lin JJ. Serratus anterior and upper trapezius electromyographic analysis of the push-up plus exercise: a systematic review and meta-analysis. J Athl Train 2019;54(11):1156-64.

11. Başkurt Z, Başkurt F, Gelecek N, Özkan MH. The effectiveness of scapular stabilization exercise in the patients with subacromial impingement syndrome. J Back Musculoskelet Rehabil 2011;24(3):173-9.

12. Ludewig PM, Cook TM. Alterations in shoulder kinematics and associated muscle activity in people with symptoms of shoulder impingement. Phys Ther 2000;80(3):276-91.

13. Kim JS, Ahn DH, Park DH, Oh JS. Electromyographic activity of the serratus anterior and pectoralis major during isometric scapular protraction at different resistance intensities in subjects with and without a winged scapula. Clin Biomech (Bristol, Avon) 2019;61:199-204.

14. Park KM, Cynn HS, Kwon OY, Yi CH, Yoon TL, Lee JH. Comparison of pectoralis major and serratus anterior muscle activities during different push-up plus exercises in subjects with and without scapular winging. J Strength Cond Res 2014;28(9):2546-51.

15. Janwantanakul P, Magarey ME, Jones MA, Dansie BR. Variation in shoulder position sense at mid and extreme range of motion. Arch Phys Med Rehabil 2001;82(6):840-4.
16. Hong J, Velez M, Moland A, Sullivan J. Acute effects of whole body vibration on shoulder muscular strength and joint position sense. J Human Kinet 2010;25(2010):17-25.

17. Ager AL, Borms D, Deschepper L, Dhooghe R, Dijkhuis J, Roy JS, et al. Proprioception: how is it affected by shoulder pain? A systematic review. J Hand Ther 2020;33(4):507-16.

18. Dilek B, Gulbahar S, Gundogdu M, Ergin B, Manisali M, Ozkan M, et al. Efficacy of proprioceptive exercises in patients with subacromial impingement syndrome: a single-blinded randomized controlled study. Am J Phys Med Rehabil 2016;95(3):169-82.

19. Hallal CZ, Marques NR, Silva SR, Dieën JV, Gonçalves M. Electromyographic activity of shoulder muscles during exercises performed with oscillatory and non-oscillatory poles. Rev Bras Fisioter 2011;15(2):89-94.

20. Ager AL, Borms D, Bernaert M, Brusselle V, Claessens M, Roy JS, et al. Can a conservative rehabilitation strategy improve shoulder proprioception? A systematic review. J Sport Rehabil 2021;30(1):136-51.

21. Abercromby AF, Amonette WE, Layne CS, McFarlin BK, Hinman MR, Paloski WH. Variation in neuromuscular responses during acute whole-body vibration exercise. Med Sci Sports Exerc 2007;39(9):1642-50.

22. Ashnagar Z, Shadmehr A, Hadian M, Talebian S, Jalaei S. The effects of whole body vibration on EMG activity of the upper extremity muscles in static modified push up position. J Back Musculoskelet Rehabil 2016;29(3):557-63.

23. Cardinale M, Lim J. Electromyography activity of vastus lateralis muscle during whole-body vibrations of different frequencies. J Strength Cond Res 2003;17(3):621-4.

24. Parry JS, Straub R, Cipriani DJ. Shoulder- and back-muscle activation during shoulder abduction and flexion using a Bodyblade Pro versus dumbbells. J Sport Rehabil 2012;21(3):26672.

25. Lister JL, Del Rossi G, Ma F, Stoutenberg M, Adams JB, Tobkin S, et al. Scapular stabilizer activity during Bodyblade, cuff weights, and Thera-Band use. J Sport Rehabil 2007;16(1):5067.

26. Suprak DN, Osternig LR, van Donkelaar P, Karduna AR. Shoulder joint position sense improves with external load. J Mot Behav 2007;39(6):517-25.

27. Park KM, Cynn HS, Yi CH, Kwon OY. Effect of isometric horizontal abduction on pectoralis major and serratus anterior EMG activity during three exercises in subjects with scapular 
winging. J Electromyogr Kinesiol 2013;23(2):462-8.

28. Boarati EL, Hotta GH, McQuade KJ, de Oliveira AS. Acute effect of flexible bar exercise on scapulothoracic muscles activation, on isometric shoulder abduction force and proprioception of the shoulder of individuals with and without subacromial pain syndrome. Clin Biomech (Bristol, Avon) 2020;72:77-83.

29. Choi WJ, Yoon TL, Choi SA, Lee JH, Cynn HS. Different weight bearing push-up plus exercises with and without isometric horizontal abduction in subjects with scapular winging: a randomized trial. J Bodyw Mov Ther 2017;21(3):582-8.

30. Criswell E. Cram's introduction to surface electromyography. Sudbury: Jones and Bartlett Publishers; 2010.

31. Sahin E, Dilek B, Baydar M, Gundogdu M, Ergin B, Manisali M, et al. Shoulder proprioception in patients with subacromial impingement syndrome. J Back Musculoskelet Rehabil 2017;30(4):857-62.

32. Allegrucci M, Whitney SL, Lephart SM, Irrgang JJ, Fu FH. Shoulder kinesthesia in healthy unilateral athletes participating in upper extremity sports. J Orthop Sports Phys Ther 1995;21(4):220-6.

33. Kim SH, Kwon OY, Kim SJ, Park KN, Choung SD, Weon JH. Serratus anterior muscle activation during knee push-up plus exercise performed on static stable, static unstable, and oscillating unstable surfaces in healthy subjects. Phys Ther Sport 2014;15(1):20-5.

34. Borstad JD, Buetow B, Deppe E, Kyllonen J, Liekhus M, Cieminski CJ, et al. A longitudinal analysis of the effects of a preventive exercise programme on the factors that predict shoulder pain in construction apprentices. Ergonomics 2009;52(2):232-44

35. Myers JB, Guskiewicz KM, Schneider RA, Prentice WE. Proprioception and neuromuscular control of the shoulder after muscle fatigue. J Athl Train 1999;34(4):362-7.

36. Escamilla RF, Yamashiro K, Dunning R, Mikla T, Grover M, Ken- niston $\mathrm{M}$, et al. An electromyographic analysis of the shoulder complex musculature while performing exercises using the Bodyblade $^{\circledR}$ Classic and Bodyblade ${ }^{\circledR}$ Pro. Int J Sports Phys Ther 2016;11(2):175-89.

37. Lee JH, Cynn HS, Kwon OY, Yi CH, Yoon TL, Choi WJ, et al. Different hip rotations influence hip abductor muscles activity during isometric side-lying hip abduction in subjects with gluteus medius weakness. J Electromyogr Kinesiol 2014;24(2):318-24.

38. Daman M, Shiravani F, Hemmati L, Taghizadeh S. The effect of combined exercise therapy on knee proprioception, pain intensity and quality of life in patients with hypermobility syndrome: a randomized clinical trial. J Bodyw Mov Ther 2019;23(1):202-5.

39. Bunton EE, Pitney WA, Cappaert TA, Kane AW. The role of limb torque, muscle action and proprioception during closed kinetic chain rehabilitation of the lower extremity. J Athl Train 1993;28(1):10-20.

40. Schulte RA, Warner C. Oscillatory devices accelerate proprioception training. Clin Biomech 2001;6(6):85-91.

41. Mörl F, Matkey A, Bretschneider S, Bernsdorf A, Bradl I. Pain relief due to physiotherapy doesn't change the motor function of the shoulder. J Bodyw Mov Ther 2011;15(3):309-18.

42. Buteau JL, Eriksrud O, Hasson SM. Rehabilitation of a glenohumeral instability utilizing the body blade. Physiother Theory Pract 2007;23(6):333-49.

43. Park W, Koo H. The effects of vibration on shoulder stabilizer muscle activation and scapular winging length of knee pushup plus exercise in adults with scapular winging. J Korean Soc Integr Med 2019;7(2):39-47.

44. Uysal Ö, Akoğlu AS, Kara D, Sezik AÇ, Çalık M, Düzgün İ. Theraband applications for improved wall slide exercise. J Athl Train 2021. [Epub]. doi: 10.4085/1062-6050-0305.21. 